\title{
X-Ray Scattering and Fluorescence from Atoms and Molecules
}

S. H. Southworth ${ }^{a}$, L. Young ${ }^{a}$, E. P. Kanter ${ }^{a}$, and T. LeBrun ${ }^{b}$

${ }^{a}$ Argonne National Laboratory, Argonne, IL 60439, USA

${ }^{b}$ National Institute of Standards and Technology, Gaithersburg, MD 20899, USA

December 3, 1998 


\section{Table of contents}

1. Introduction

2. Photon-atom interactions in the $\mathrm{x}$-ray regime

3. Photon scattering from free atoms

4. Subshell-specific Compton scattering: infrared divergence

5. Inelastic scattering probes of electron pair correlation and other properties

6. Experimental methods for x-ray fluorescence spectroscopy

7. X-ray resonant Raman scattering from atoms

8. X-ray polarization anisotropy of aligned molecules

9. Nondipole x-ray resonant Raman scattering from molecules

10. Acknowledgements

11. References

12. Figure captions 


\section{Introduction}

Fundamental understanding of $\mathrm{x}$-ray interactions with atoms and molecules provides a basis for applying $\mathrm{x}$-ray methods to complex materials, such as structural determinations by $\mathrm{x}$-ray diffraction and extended $\mathrm{x}$-ray absorption fine structure, Compton scattering measurements of electron-momentum distributions, and determinations of local chemical environments by $\mathrm{x}$-ray absorption near-edge spectroscopy. This report discusses the basic physics of $\mathrm{x}$-ray interactions with atoms and molecules relevant to understanding elastic and inelastic scattering cross sections far from threshold (Sections 3-5) and resonance and threshold phenomena in x-ray fluorescence (Sections 7-9). The ideas are discussed in the context of providing theoretical interpretations of experimental results and are topics of ongoing theoretical developments. We also discuss experimental methods of $\mathrm{x}$-ray spectroscopy using solid-state and wavelength-dispersive spectrometers with excitation by tunable synchrotron radiation. The examples discussed are primarily from the authors' research. We did not attempt to provide a comprehensive review, but several recent review articles and research papers are referenced for further information. The ideas discussed are general and relevant to understanding fundamental studies and applications of $\mathrm{x}$-ray scattering and fluorescence.

\section{Photon-atom interactions in the $x$-ray regime}

The primary photon-atom interactions at $\mathrm{x}$-ray energies are photoabsorption and elastic and inelastic scattering, ${ }^{1}$ as illustrated in Fig. 1 for a high- and low-Z element. For incident energies below the K-shell ionization threshold, photoabsorption dominates as in the visible and ultraviolet energy ranges. However, with increasing energy, the photoabsorption cross section falls as $\mathrm{E}^{-7 / 2}$, and, especially for low-Z elements, scattering becomes dominant over a large energy range until the onset of pair production and nuclear resonant processes at $\mathrm{MeV}$ energies. Photoabsorption, elastic (Rayleigh) and inelastic (Compton) scattering are the lowest-order interactions between the radiation field and an electron in an atom. The non-relativistic interaction Hamiltonian is of the form 


$$
H_{\mathrm{int}}=\frac{e^{2}}{2 m c^{2}} \mathbf{A}^{2}-\frac{e}{m c} \mathbf{p} \bullet \mathbf{A}
$$

where $\mathbf{p}$ is the electron momentum and $\mathbf{A}$ is the vector potential of the electromagnetic field. Photoabsorption, accompanied by photon anniliation, is described by the $\mathbf{p} \cdot \mathbf{A}$ term in the expression. Scattering, however, is a process which conserves photons, and $\mathbf{A}$ therefore must appear an even number of times. Scattering processes (elastic and inelastic) are therefore described in lowest order by the $\mathbf{A}^{2}$ term. These, as well as higher-order scattering processes, i.e. second-order $\mathbf{p} \cdot \mathbf{A}$ processes, can be visualized by the Feynmann diagrams in Fig. 2. The first-order $\mathbf{A}^{2}$ scattering is the "seagull" diagram (Fig. 2a) and dominates in regions far from threshold and resonance (Section 3). The second and third diagrams result from second-order $\mathbf{p} \cdot \mathbf{A}$ interactions, with emission first (Fig. $2 b$ ) and absorption first (Fig. 2c). The diagrams account for infrared divergence (Section 4) and resonant Raman scattering (Sections 7-9). ${ }^{2}$ The corresponding equation is known as the Kramers-Heisenberg formula. ${ }^{3}$

\section{Photon scattering from free atoms}

In contrast to the photoabsorption process, relatively little data exists on the scattering of photons from free atoms. The reason for this is clear upon inspection of Fig. 1, where it is seen that the cross section for scattering is substantially smaller than that for photoabsorption, being on the order of the Thomson cross section for the scattering from a free electron, $\square_{T}=8 \square \mathrm{r}^{2 / 3}=0.6652 \times 10^{-24} \mathrm{~cm}^{2}$. The coherent (or Rayleigh) and incoherent (or Compton) scattering processes are commonly described (within the $\mathbf{A}^{2}$ approximation) by modifying the scattering from a point charge to account for the extended charge distribution through the use of form factors and incoherent scattering factors, $F(k)$ and $\mathrm{S}(\mathrm{k}) .{ }^{4}$ For an unpolarized beam

$$
\left(\frac{\mathrm{d} \square}{\mathrm{d} \square}\right)_{\mathrm{coh}}=\left(\frac{\mathrm{d} \square \mathrm{T}}{\mathrm{d} \square}\right)|\mathrm{F}(\mathrm{k})|^{2}=\frac{\mathrm{r}_{\mathrm{e}}^{2}}{2}\left(1+\cos ^{2} \square\right)|\mathrm{F}(\mathrm{k})|^{2}
$$


and

$$
\left(\frac{\mathrm{d} \square}{\mathrm{d} \square}\right)_{\mathrm{inc}}=\left(\frac{\mathrm{d} \square \mathrm{KN}}{\mathrm{d} \square}\right) \mathrm{S}(\mathrm{k})=\frac{\mathrm{r}_{\mathrm{e}}^{2}}{2}\left(\frac{\square_{2}}{\square_{1}}\right)^{2}\left[\frac{\square_{1}}{\square_{2}}+\frac{\square_{2}}{\square_{1}}-\sin ^{2} \square\right] \mathrm{S}(\mathrm{k})
$$

Here, $\hbar \mathrm{k}=\hbar\left(\mathrm{k}_{2}-\mathrm{k}_{1}\right)$ and $\hbar \square=\hbar\left(\square_{2}-\square_{1}\right)$ are the momentum and energy transferred in the scattering, and $\square$ is the angle between incident and outgoing photons. The $F(k)$ and $S(k)$ are given by, ${ }^{4}$

$$
\mathrm{F}(\mathrm{k})=\sum_{\mathrm{n}=1}^{\mathrm{Z}}\left\langle i\left|\exp \left(\mathbf{i k} \cdot \mathbf{r}_{\mathrm{n}}\right)\right| i\right\rangle
$$

and

$$
\mathrm{S}(\mathrm{k})=\sum_{\mathrm{m}=1}^{\mathrm{Z}} \sum_{\mathrm{n}=1}^{\mathrm{Z}}\left\langlei \left|\operatorname { e x p } \left(\mathrm{ik} \cdot\left(\mathbf{r}_{\mathrm{m}}-\mathbf{r}_{\mathrm{n}}\right)|i\rangle-|\mathrm{F}(\mathrm{k})|^{2}\right.\right.\right.
$$

The form factor approach is important for several reasons. Essentially all tabulations of elastic scattering use this approximation to estimate the Rayleigh scattering contribution. ${ }^{5}$ Both $F(k)$ and $S(k)$ can be evaluated in terms of ground state wavefunctions and are conveniently tabulated for theoretical calculations of varying complexity. ${ }^{6}$ Cross sections are conveniently defined as a function of only one variable, the momentum transfer $\mathrm{k}$. Finally, physical interpretations can be given to the measured quantities: the elastic scattering form factor $F(k)$ is the Fourier transform of the charge density, and the inelastic scattering factor $S(k)$ is related to the electron pair distribution function (see Section 5). Through the impulse approximation, its doubly differential counterpart $S(k, \square)$ yields the

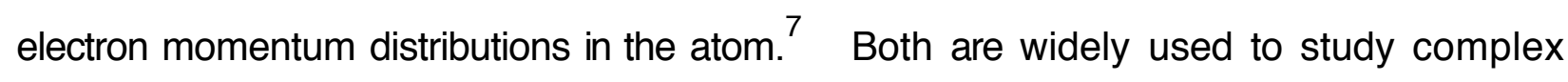
materials ${ }^{8}$ and charge density distributions. ${ }^{9}$ However, the range of validity of the form factor approach is limited at low energies due to the neglect of binding, and, at high energies and large angles, due to the assumption of low momentum transfer $\hbar \mathrm{k}<\mathrm{mc}^{10}$

Theoretical approaches that go beyond the form factor and incoherent scattering factor have been developed recently. For a review of these second-order S-matrix calculations 
of Rayleigh scattering see Kissel et al. ${ }^{11}$ and for Compton scattering see Bergstrom and Pratt. $^{2}$ They are generally considered state-of-the-art, and treat both the $\mathbf{A}^{2}$ and the second-order $\mathbf{p} \cdot \mathbf{A}$ terms simultaneously in a fully relativistic framework. For Rayleigh scattering, the additional dynamical terms correspond to the anomalous scattering factors, which alter the phase and magnitude of the scattered photon field due to absorption in the atom. For Compton scattering these second-order terms correspond to Raman scattering and the infrared divergence. These general methods which treat the interaction to higher order require considerable computational effort, and necessarily treat the atomic wavefunctions using the independent particle approximation (IPA). It is of interest to understand the improvements relative to simpler methods which are able to treat the target more accurately.

Discrepancies between the S-matrix calculations and tabulations based on other theories are fairly substantial in light elements, even at energies high enough where the S-matrix and the tabulations agree in higher-Z elements. Only four early experiments exist for scattering from free atoms, ${ }^{12-15}$ the case for which cross sections are usually calculated. Wollan ${ }^{12}$ measured total scattering in various gases relative to $\mathrm{H}_{2}$. Experimental uncertainties at this early stage of instrumentation made comparison with theory difficult. ${ }^{14}$ Chipman and Jennings ${ }^{13}$ carefully measured absolute total scattering cross sections for rare gases at 17.4 $\mathrm{keV}$ and angles $<32^{\circ}$ and confirmed form factor calculations using then-current theory to estimate the inelastic component. Ice et al. ${ }^{14}$ using synchrotron radiation, measured total cross sections in $\mathrm{H}_{2}$ and $\mathrm{He}$, and found evidence for electron correlation effects. Smend and Czerwinski ${ }^{15}$ focused on the large angle elastic scattering in $\mathrm{Kr}$ and $\mathrm{Xe}$ at $59.54 \mathrm{keV}$, finding better agreement with the S-matrix than with form factor calculations. In light of recent theoretical developments, ${ }^{2}$ it is of general interest to understand the regions of validity for the various calculational methods and develop generalized schemes for the prediction of 
scattering cross-sections, angular distributions, and polarization dependencies. In an initial experiment at Argonne's Advanced Photon Source (APS), Compton and Rayleigh scattering from $\mathrm{Ne}$ were measured between 11 and $22 \mathrm{keV}$, where sizable differences (up to $16 \%$ ) existed between the S-matrix and form-factor calculations of the elastic scattering channel. $^{16}$

Early measurements did not resolve the Compton and Rayleigh components, ${ }^{12-14}$ making detailed comparison with theory difficult. Two modern advances enable higher quality scattering data: 1) high incident photon flux at synchrotron sources coupled with highresolution crystal monochromators enables gas-phase experiments, and 2) advances in theoretical methods permit scattering cross sections to be placed with high precision $(\approx 0.5 \%)$ on an absolute scale by comparison to well-known cross sections for $\mathrm{He}$. Although crystal spectrometers provide significantly better resolution (e.g. $\Delta \mathrm{E} / \mathrm{E} \approx 10^{-4}$ for Si (111)), there are advantages to using relatively low-resolution $(\Delta E / E \approx 3 \%)$ solid state detectors in some situations. First, solid state detectors, such as $\mathrm{Si}(\mathrm{Li}) \mathrm{s}$, permit simultaneous data collection over the entire usable energy range ( $\mathrm{Si}(\mathrm{Li}) \mathrm{s}$ have efficiencies $>10 \%$ from $\approx 1$ to $60 \mathrm{keV}$ ) as opposed to the small energy window available using crystal spectrometers. Second, because of their high efficiencies, absolute scattering cross sections can be obtained simply using well-characterized solid state detectors to make measurements relative to $\mathrm{He}$ and using theory to put values on an absolute scale. Third, solid state detectors, being compact, can be arranged to subtend large solid angles for efficient coincidence experiments.

For this first experiment, only ratios are measured: 1) total scattering in $\mathrm{Ne}$ to $\mathrm{He}$, and 2) Compton to Rayleigh scattering in $\mathrm{Ne}$, thus circumventing several difficult absolute measurements, e.g. x-ray flux and detector efficiency. In the energy range 11-22 keV, which is far above the Ne K-edge $(870 \mathrm{eV})$, the Compton and Rayleigh scattering cross sections are comparable and the IPA may be reasonably well-justified. The total cross section for $\mathrm{x}$-ray scattering from $\mathrm{He}$ in this energy range is dominated by the Compton 
channel, with the Rayleigh contribution ranging from $\approx 5-0.1 \%$. Calculations for $x$-ray scattering cross sections from $\mathrm{He}^{4-6}$ are in good agreement and are assumed to be correct.

In order to compare measured cross sections with theory, it is important to eliminate uncertainties due to the polarization of the incident $x$-ray beam and variations therein during the measurement process. This can be done using the configuration shown in Fig. 3, which measures simultaneously the scattering yield in two detectors placed $90^{\circ}$ azimuthally about the photon beam propagation axis. The average yield in the two detectors is the polarization-independent differential scattering cross section. Monochromatic $\mathrm{x}$-rays incident on the gas sample create a line source viewed by the two well-characterized $\mathrm{Si}(\mathrm{Li})$ detectors. Higher harmonics in the incident $\mathrm{x}$-ray beam are not a direct factor here, since they are resolved in the $\mathrm{Si}(\mathrm{Li})$ detectors, but can be monitored using ion chambers and suitable attenuators.

An example of the scattering data is shown in Fig. 4, which shows an empty cell spectrum followed by spectra from $\mathrm{Ne}$ at $11,15,18$, and $22 \mathrm{keV}$. The empty cell spectrum is featureless (no window scattering) and serves as a good measure of background. After background subtraction, the yields were corrected for detector livetime, window and gas attenuation, $x$-ray intensity and pressure dependence. The $\approx 1 \%$ errors associated with these early measurements of the total scattering cross sections are composed of: statistical error $(\approx 0.2 \%)$ and systematic errors due to 1$)$ variations in background $(\approx 0.2 \%), 2$ ) extrapolation to zero pressure $(\approx 0.4 \%)$, and 3$)$ run-to-run variation in He normalization $(\approx 0.7 \%)$. Reductions in systematic errors are expected in a double-cell arrangement where both the He reference gas and the Ne sample gas can be measured simultaneously.

Separation of the Compton and Rayleigh components in the spectra requires detailed knowledge of both the instrumental response and the lineshape associated with the Compton scattering. For a $\mathrm{Si}(\mathrm{Li})$ detector operated in this energy range the response to a monochromatic $x$-ray input can be modeled as a Gaussian peak with a minor $(\approx 0.5 \%)$ lowenergy exponential tail and a small $(\approx 0.1 \%)$ escape peak. The detailed response of the 
detectors can be determined through calibration using radioactive sources. ${ }^{17}$ However, the most reliable measurements come from an in situ calibration using, e.g., Compton scattering from $\mathrm{He}$ or fluorescence from $\mathrm{Kr}$. The in situ measurements are important in that they quantify the width of the Gaussian response during the experiment. The Gaussian peak standard deviation is characterized by $\square^{2}=a+b E$, where $E$ is the incident photon energy and $a$ and $b$ are calibration constants. It was found that the lineshape associated with Compton scattering can be modeled adequately in the peak region using impulse approximation Compton profiles ${ }^{18}$ in a Monte-Carlo simulation ${ }^{17}$ (see Fig. 5). In using the tabulated Compton profiles to fit the data, it is extremely important to include kinematic cutoffs for each subshell (see Figs. 6 and 7). The amplitudes and positions of the generated Compton and Rayleigh lineshapes were varied in a four-parameter fit to the experimental data (see Fig. 8). While the overlap between the Compton and Rayleigh scattering is substantial at $11 \mathrm{keV}$, the separation is reliable to $\approx 8 \%$. The error in the Compton/Rayleigh ratio decreases to $\approx 2 \%$ at $22 \mathrm{keV}$.

With these precise measurements of ratios (total scattering from $\mathrm{Ne}$ relative to $\mathrm{He}$ and Compton to Rayleigh scattering from $\mathrm{Ne}$ ), detailed comparisons with theory are feasible. The analysis reveals the importance of including electron exchange and electron correlation in the atomic wavefunctions and the dynamical-scattering effects (second order $\mathbf{p} \cdot \mathbf{A}$ terms) in accurate calculations of scattering cross sections for low-Z atoms. Since no theory to date includes all of these effects simultaneously, a perturbative method was prescribed to obtain predictions with $1 \%$ accuracy. ${ }^{16}$

Outstanding challenges in this area of research are 1) understanding the limitations of this perturbative correction method at lower momentum transfers, where cross-terms between the largest corrections, the exchange (10\%) and dynamical effects (5\%), will increase; 2$)$ understanding the angular dependence of the anomalous scattering factors, where the common assumption is angle independence, but $\approx 40 \%$ differences have been predicted $^{19}$; 3) understanding and developing theoretical models for anomalous scattering 
near absorption edges. ${ }^{20}$ Research of this type impacts a number of fields, because form and structure factors are widely used for structure determination and radiation transport calculations. Yet no single theory is presently adequate, due to the need to include both the x-ray interaction and the atomic wavefunction accurately.

\section{Subshell-specific Compton scattering: infrared divergence}

The previous section focused on the inelastic scattering spectrum near the Compton peak. In this section, the focus is on the inelastic scattering cross section $\mathrm{d}^{2} \square / \mathrm{d} \square \mathrm{d} \square$ at low energy, which has been predicted to diverge as $1 / \square$, as $\square \square 0$, where $\square$ is the scattered photon energy. The implication of an inelastic scattering spectrum which is infrared divergent is that the cross section is undefined unless a lower energy limit to the detection system is specified. The theoretical situation is clear, with considerations on general grounds, ${ }^{21}$ nonrelativistic calculations, ${ }^{22}$ and relativistic S-matrix calculations ${ }^{23}$ all in agreement about the existence of an infrared divergence (IRD). However, the experimental situation is not as clear. There is not, to date, an unambiguous observation of the onset of the infrared rise. Early reports of an infrared divergent rise for scattering from the L-shell of $\mathrm{Zr}^{24}$ were questioned by Marchetti and Franck ${ }^{25}$ who suggested that the observed rise could be due to interatomic bremsstrahlung following photoeffect. Manninen et al. ${ }^{26}$ later observed an infrared divergent rise, but concluded that it was due entirely to photoelectron bremsstrahlung in the target. The magnitude of bremsstrahlung is a function of the thickness of the target, and therefore questions have been raised as to the correct procedure for its subtraction. ${ }^{23}$ All of the studies above were performed on solid targets, motivating our use of a thin gas target in order to minimize the interatomic bremsstrahlung correction.

The approach taken for this experiment was to isolate the scattering from the K-subshell in gaseous Ar, where the predicted infrared rise of the cross section was accessible to the energy range of $\mathrm{Si}(\mathrm{Li})$ detectors. Compton scattering from the $\mathrm{K}$-shell of $\mathrm{Ar}$ was identified 
by detecting the $\mathrm{K} \square, \square$ fluorescence photon in coincidence with the Compton-scattered photon. The layout of the experiment is shown in Fig. 9. In this view, the incident x-ray beam is normal to the page and traverses Ar gas which fills the region between the detectors. Monochromatic $40 \mathrm{keV} x$-rays from the bending-magnet beamline at the Basic Energy Sciences Synchrotron Radiation Center of the APS were incident on the Ar target. At $40 \mathrm{keV}$, the ratio of photoabsorption to Compton scattering in $\operatorname{Ar}$ is $\approx 7$, and the fluorescence quantum yield is $\approx 12 \%$, therefore the relative photon yields are well-matched for the two processes. Since the incident $x$-rays are polarized in the horizontal plane, the Compton scattering is maximized in the vertical direction toward $\mathrm{Si}(\mathrm{Li}) 1$ and $\mathrm{Si}(\mathrm{Li}) 2$ which have the largest solid angles. During the experiment, the energies and relative arrival times of photons hitting $\mathrm{Si}(\mathrm{Li}) \mathrm{s} 1,2$, and 3 were recorded in "event" mode. This permits resorting the data after the experiment to further explore the coincidences and correlations between all three detectors. Secondary scattering into the detectors was minimized by lead shielding, and photoelectron penetration into the detectors was reduced by placing kapton film over the detector entrance apertures.

A spectrum of the difference in the arrival times of photons in Detectors 1 and 2 is shown in Fig. 10. The large spike at zero time corresponds to the "prompt" coincidences, where both detectors simultaneously detect photons, but contains both "true" and some "random" events. The remainder of the spectrum is completely due to random coincidences exhibiting the complex time structure of the APS storage ring. "True" coincidences are defined as (prompt - random) coincidences. The periodicity of the broad envelope is due to the revolution time of a single pulse in the storage ring $(\approx 3.6 \square \mathrm{s})$, and the fine structure corresponds to the $\square 100$ ns between filled buckets.

The correlated energy spectra in Detectors 1 and 2 within the $t=0$ peak are shown in two dimensions in Fig. 11a. Making the appropriate cuts on the $\mathrm{K} \square, \square$ line $(\approx 3 \mathrm{keV})$ in Detectors 1 and 2 will yield the inelastic scattering spectrum from the K-shell. The K $\square, \square$ lines are only faintly visible in this view, and correspond to horizontal and vertical lines starting from the spot near the lower left corner. It is clear from Fig. 11a that substantial 
contamination of the true coincidences is present. The contamination is due primarily to detector-detector scattering in this particular pair of detectors. For example, the diagonal lines extending from $\approx 40 \mathrm{keV}$ from both axes corresponds to the constant sum-energy line. True coincidences along this line can originate from Rayleigh scattering off the target into Detector 1 and subsequent Compton backscattering into Detector 2, leading to the sharp diagonal line. A similar explanation holds for the diffuse spots, which originate from Compton scattering from the target. These crosstalk events vanish in coincidences of Detectors 1 or 2 with the horizontal Detector 3 (see Fig. 11b).

Figure 12 shows the resulting true coincidence spectrum after the appropriate time and energy cuts on the data have been made. Overlaid as a solid line is the singles spectrum for comparison. The Rayleigh line is offscale at $40 \mathrm{keV}$. The full-atom Compton spectrum peaks around $37 \mathrm{keV}$ and is dominated by scattering from outer shells with narrower electron-momentum distributions. A comparison of the coincidence spectrum with an impulse approximation ${ }^{18} \mathrm{~K}$-shell profile is in reasonable agreement, as shown in Fig. 13. The rise at low energy is somewhat larger than predicted for infrared divergence, and is probably mostly photoelectron bremsstrahlung. An improved experiment, with better detector geometry and pressure-dependent measurements, is necessary to isolate the IRD. However, the excess in the coincidence spectrum at the $\mathrm{K} \square, \square$ energy $(\square 3 \mathrm{keV}$ ) is interesting in that it suggests the creation of a double-K vacancy state. This observation has led to further studies of the cross section for double-K vacancy production which will be reported elsewhere.

Of general interest are the analyses of pulsed synchrotron sources for inelastic scattering studies using coincidence techniques. ${ }^{27,28}$ Although the fluxes from synchrotron sources are extremely high relative to radioactive sources, the measurement time for a given signal-tonoise ratio is not significantly reduced. This results from the fact that the true coincidence rate is proportional to where $I$ is the incident $x$-ray intensity, $\square_{1}$ and $\square_{2}$ are the detection efficiencies for the 
scattered and fluorescence photon, and $\square$ is the time resolution of the detector system. Therefore, the ratio of true-to-random events is maximized as $I \square 0$, so the high intensity from third-generation synchrotron sources is not necessarily utilized during the measurement process, albeit being extremely useful during setup of the experiment. The principal advantage of these new sources for such experiments lies in the tunability and narrow bandwidth of the beam.

\section{Inelastic scattering probes of electron pair correlation and other properties}

High energy $\mathrm{x}$-ray scattering is regarded as an effective probe of target properties, because the photon-atom interaction is impulsive, allowing the first Born $\left(\mathbf{A}^{2}\right)$ approximation to be made. $^{29}$ With this approximation, cross sections are factored into two terms, dealing with the projectile and the target separately. Target properties are contained in the generalized oscillator strength (GOS) $\frac{d f(\mathbf{k}, \square)}{d \Pi}$,

$$
\frac{d f(\mathbf{k}, \square)}{d \square}=\frac{\square}{R\left(\mathrm{ka}_{0}\right)^{2}} \sum_{f}\left|\left\langle f\left|\sum_{\mathrm{n}=1}^{\mathrm{Z}} \exp \left(\mathbf{i k} \cdot \mathbf{r}_{\mathrm{n}}\right)\right| i\right\rangle\right|^{2}
$$

where $\mathbf{k}$ and $\square$ are the momentum and energy transferred in the scattering process. Here, T $\mid$ and $\mid \mathrm{i} \square$ are the final and initial states of the target atom with $\mathbf{Z}$ electrons, $\mathbf{r}_{\mathrm{n}}$ the radial vector from the nucleus of the $\mathrm{n}^{\text {th }}$ electron, $\mathrm{a}_{\mathrm{o}}$ the Bohr radius, and $R$ the Rydberg constant. The GOS is closely related to the dynamic structure factor, $S(\mathbf{k}, \square)$, and the doublydifferential inelastic scattering spectrum, $\left(\mathrm{d}^{2} \square / \mathrm{d} \square \mathrm{d} \square\right)$. The Bethe surface (a threedimensional representation of $d f(\mathbf{k}, \square) / d \square$ as a function of $\ln \left(\mathrm{ka}_{0}\right)^{2}$ and $\left.\square\right)$ gives the best visualization of the GOS. Complete studies of any Bethe surface using inelastic x-ray scattering are difficult due to the small cross section and the need to reliably remove the intense elastic scattering peak. Some studies have been done using $\mathrm{x}$-ray scattering, ${ }^{30}$ but most have used electron scattering on gaseous targets. ${ }^{31}$ Many properties can be 
calculated from the GOS, e.g. polarizability, dielectric response, and the electron pair correlation function. $^{32}$

Here the focus is on the static structure factor, $S(k)$, and its well-known sensitivity to electron correlation. $^{5}$ Originally outlined by Waller and Hartree in 1929, the total (elastic plus inelastic) scattering cross section can be inverted to yield the electron pair distribution function, $\mathrm{h}\left(\mathbf{r}_{12}\right)$, the radial probability of finding an electron $\mathbf{r}_{12}$ away from another. This can be easily seen by rearrangement of the following expression for $S(k)$,

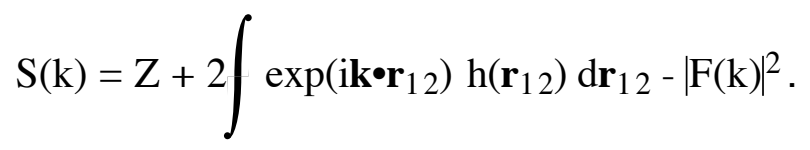

The pair distribution is strongly affected by Coulomb correlation and therefore measurements of total scattering cross sections can, in principle, discern IPA from models including correlation. This was demonstrated on $\mathrm{H}_{2}$ and $\mathrm{He}$ in the first study using synchrotron radiation $^{14}$ and in others using $x$-ray tubes. ${ }^{33}$ Bonham $^{34}$ pointed out that the interpretation of these results was based upon a simplified theory, where the questionable issue is that doubly-differential cross sections are commonly measured at fixed-angle and not fixed momenum transfer as required by Waller-Hartree theory. He derived formulae for $(\mathrm{d} \square / \mathrm{d} \square)_{\text {inel }} \mu \mathrm{S}(\mathrm{k})$, where the quantity $\left(\mathrm{d}^{2} \square / \mathrm{d} \square \mathrm{d} \square\right)_{\text {inel }}$ was integrated over energy transfer for the cases of constant scattering angle and constant momentum transfer.

Recently, measurements of $S(k)$ have been obtained by integrating the energy- and angle-resolved $x$-ray inelastic scattering spectrum on a variety of liquids after correction for background, detector efficiency, and elastic scattering. ${ }^{35}$ The absolute scale was then determined using the Bethe sum rule. ${ }^{29}$ The variation of the value of the momentum transfer over the spectra was ignored in the analysis. Comparison of $S(k)$ to theoretical calculations using wavefunctions varying in complexity from the simplest independent atom model (IAM) to the configuration interaction singles and doubles (CISD), shows markedly improved agreement when both electron correlation and chemical binding effects are 
included. However, discrepancies of order $10 \%$ remain. Various sources of these discrepancies were suggested: breakdown of the first Born approximation, neglect of molecular vibrations, and relativistic effects. The studies described in Section $3^{16}$ show that corrections to cross sections calculated by the simplest $A^{2}$ theory, i.e. first Born approximation, can be substantial. Refined measurements to determine $S(k)$ in gases are currently in progress at Argonne. These are expected to reveal the relative importance of the corrections which should be applied to calculations using the $\mathbf{A}^{2}$ approximation.

\section{Experimental methods for $\mathrm{x}$-ray fluorescence spectroscopy}

Ejection of a deep inner-shell electron by photoabsorption or particle impact produces a vacancy state which rapidly relaxes $\left(\left[10^{-15} \mathrm{~s}\right)\right.$ by a radiative (x-ray fluorescence) or radiationless (Auger electron emission) process. Here we will focus on $\mathrm{x}$-ray fluorescence spectra (XFS) recorded with wavelength-dispersive spectrometers to resolve electronic states. Characteristic XFS can be excited by particle impact or non-resonant $\mathrm{x}$-ray absorption using laboratory sources and has long been studied. ${ }^{36}$ However, we focus here on variations of XFS when tunable synchrotron radiation is used to excite inner-shell electrons to Rydberg or valence-like resonant states or to low-energy continuum states near the ionization threshold. The photoexcitation and decay processes are coupled at innershell resonances and thresholds, producing several interesting phenomena in XFS of atomic, molecular, and solid-state samples. ${ }^{37,38}$ We begin with a discussion of experimental methods.

Deslattes et al. ${ }^{39}$ performed pioneering experiments on Ar K $\square$ XFS which demonstrated the potential of tunable synchrotron radiation for studies of inner-shell threshold phenomena. Subsequently, a beamline dedicated to $x$-ray spectroscopy in the energy range $\square 1-5 \mathrm{keV}$ was developed at Brookhaven's National Synchrotron Light Source. ${ }^{40}$ Several XFS experiments have been performed on atoms, molecules, and solids using a curved-crystal 
spectrometer with position-sensitive detector. ${ }^{41} \mathrm{~A}$ diagram of the instrumentation used for gas-phase studies is shown in Fig. 14. The beamline ${ }^{40}$ uses a bending-magnet source of synchrotron radiation, followed by a collimating mirror, double-crystal monochromator, and focusing mirror. Using $\mathrm{Si}(111)$ crystals in the double-crystal monochromator ${ }^{42}$ typically provides a flux $\square 10^{11} \mathrm{~s}^{-1}$ at $3.2 \mathrm{keV}$ (the Ar K-edge) at a bandwidth of $\square 0.8 \mathrm{eV}$.

The focused x-ray beam passes through a target gas cell, and fluorescent $\mathrm{x}$-rays emitted normal to the incident $x$-ray beam are dispersed by a curved crystal ${ }^{43}$ and detected by a position-sensitive proportional counter (PSPC). ${ }^{44}$ Thin windows of low-Z material, such as $13 \square \mathrm{m}$ polypropylene, are used to contain the sample gas while providing high transmission of $\mathrm{x}$-rays into and out of the gas cell. lonization chambers or thin-foil photocurrent detectors are used to measure the intensities of the incident and transmitted $x$ ray beam, providing absorption spectra and normalization for variations in beam intensity. The incident $x$-rays pass through a path length of $\square 10 \mathrm{~mm}$ of sample gas, and the pressure (口100 torr) is adjusted to absorb $30-50 \%$ of the incident $x$-rays. This produces an extended source of fluorescent x-rays accepted by the curved crystal. By placing the target cell inside the Rowland circle defined by the crystal and detector and using a positionsensitive detector, a range of $x$-ray energies is recorded simultaneously. ${ }^{41}$ Typically, the acceptance window of the PSPC is wide enough to simultaneously record the entire XFS of interest for a given experiment, so there is no need to normalize the recorded spectrum for variations in the intensity of the incident $\mathrm{x}$-ray beam. Parallel recording of the XFS is crucial, since the total counting rates are typically $10-100 \mathrm{~s}^{-1}$. Once the spectrometer angle is set to record a given XFS, the sample gas is replaced with $\mathrm{N}_{2}$ to measure the dispersion and sensitivity across the PSPC window by elastic $x$-ray scattering. The target cell is then refilled with sample gas, and variations in the XFS are studied as the incident X-ray energy is scanned through resonances and threshold. 
The small collection solid angle of the spectrometer is compensated by use of large sample gas densities to absorb a large fraction of the incident $x$-rays. Such high sample pressures are incompatible with electron or ion spectroscopy but are suitable for x-ray spectroscopy. Since the energies of the $x$-ray fluorescence lines are shifted down from the energy of the inner-shell absorption edge, absorption of the fluorescent $\mathrm{x}$-rays by the sample gas is due to shallower subshells (at energies far above their edges) and is much smaller $(\square 10 \%)$ than for the incident $x$-rays. Although the results are not discussed here, measurements of resonant elastic $x$-ray scattering ${ }^{45}$ are sensitive to absorption in the gas cell, so the path length of scattered $x$-rays through the sample gas is minimized, and the pressure dependence of the scattered intensities is measured. A related issue is the possibility of quenching the vacancy states due to collisions with other sample gas molecules. However, the mean time between collisions for a sample gas at 760 torr and $15^{\circ} \mathrm{C}$ is $\square 10^{-10} \mathrm{~s},{ }^{46}$ much longer than the $\square 10^{-15} \mathrm{~s}$ of an $\mathrm{x}$-ray fluorescence process, so collisional effects are negligible.

Synchrotron radiation from a bending magnet source is highly linearly polarized when viewed along the stored-electron orbital plane with a narrow vertical acceptance. ${ }^{47}$ For the beamline in Ref. 40 , 95\% linear polarization is calculated for typical use of \pm 80 पrad vertical acceptance. The degree of linear polarization is enhanced by the double-crystal monochromator when the Bragg angles are near $45^{\circ}$. The linear-polarization selectivity of the curved-crystal spectrometer can also be quite high. ${ }^{41}$ In particular, several polarizationdependent studies have been made on $\mathrm{Cl}$-containing molecules of $\mathrm{Cl} \mathrm{K}-\mathrm{V}$ x-ray emission, in which a valence electron fills a vacancy in the $\mathrm{Cl} \mathrm{K-shell.}{ }^{48-52}$ For incident $\mathrm{x}$-ray energies near the Cl K-edge ( $\square 2820-2880 \mathrm{eV})$ and for the $\mathrm{Cl} \mathrm{K}-\mathrm{V}$ x-ray fluorescence $(\square 2800-2820$ $\mathrm{eV})$, the Bragg angles for $\mathrm{Si}(111)$ crystals are $\square 43^{\circ}-45^{\circ}$, giving essentially complete polarization selectivity. Polarization-selective measurements have also been made on $\mathrm{S}$ containing molecules ${ }^{53,54}$ and on atomic Ar. ${ }^{55,56}$ The curved-crystal spectrometer in Fig. 14 
can be rotated about the emitted $\mathrm{x}$-ray direction to measure the fluorescence polarization, ${ }^{48}$ or it can be rotated about the incident $\mathrm{x}$-ray beam direction to measure the angular distribution of polarized fluorescence. ${ }^{51}$ Such measurements give information on the spatial alignment of resonantly-excited molecules and on the symmetries of the electronic states (Section 8).

We have recently performed $\mathrm{x}$-ray spectroscopy experiments using $012-14 \mathrm{keV} \mathrm{x}$ rays on a high-brightness undulator beamline at Argonne's APS. The beamline uses a double-crystal monochromator ${ }^{57}$ with a liquid-nitrogen-cooled, thin $\mathrm{Si}(111)$ crystal $^{58}$ which maintains resolution and brightness despite the high power density of the undulator beam. The beamline has been used for a variety of experiments over the energy range $\square 3-28$ $\mathrm{keV}$, and provides a flux $\square 10^{13} \mathrm{~s}^{-1}$ in a bandwidth $\Delta \mathrm{E} / \mathrm{E} \square 1.8 \times 10^{-4}$. X-ray fluorescence experiments were performed on the $\mathrm{K}$-shells of $\mathrm{Kr}$ and $\mathrm{Br}_{2}$ using the spectrometer system shown in Fig. 15. Beamline x-rays pass through the target gas cell with ion chambers front and back to measure the intensities of the incident and transmitted beam. An incident x-ray beam passing through a gas target typically produces a line-like source of fluorescent $x$ rays, and the spectrometer was optimized for this geometry. A 5-10 $\mathrm{mm}$ line of $x$-ray fluorescence produced in the sample cell was collimated with apertures, dispersed by two flat $\mathrm{Si}(111)$ crystals oriented in high-resolution dispersive geometry, and detected by a $\mathrm{Nal}$ scintillator. The crystals are positioned on precision translational and rotary stages under computer control to allow more complex experimental geometries in the future, including curved-crystal scans along the Rowland circle. In the present geometry, x-ray spectra were recorded by step-scanning the first crystal with the second crystal fixed. This varies the Bragg angle defined by the two-crystal system at one-half the angular speed as the included angle. A double-flat-crystal system was chosen for initial experiments to allow the resolution to approach the theoretical limit for $\mathrm{Si}(111)\left(\Delta \mathrm{E} / \mathrm{E}=1.41 \times 10^{-4}\right){ }^{59}$ However, the resolution was limited by the $\square 4 \mathrm{~mm}$ width of the incident $\mathrm{x}$-ray beam, which was used 
without focusing in early experiments. The initial results obtained on resonance and threshold effects at $\mathrm{Kr}$ and $\mathrm{Br}_{2} \mathrm{~K}$-shells complement studies at lower $\mathrm{x}$-ray energies made with the instrumentation in Fig. 14. However, higher resolution is needed to resolve valence molecular orbitals in K-V XFS such as for $\mathrm{Br}_{2}$ at $13.4 \mathrm{keV}$. Future versions of the $\mathrm{x}$-ray spectrometer could use a curved crystal and position-sensitive detector to increase efficiency, but with a high-resolution reflection such as $\mathrm{Si}(333)$. Beamline focusing mirrors will also be used to produce an intense, narrow source of fluorescent x-rays in the target gas cell.

\section{X-ray resonant Raman scattering from atoms}

When an atomic inner-shell vacancy is produced by particle impact or photoionization far above threshold, the resulting XFS has characteristic energies, intensities, and line shapes. A two-step model, in which the vacancy creation process and radiative decay process are treated independently, is adequate to describe the XFS. However, when vacancies are produced by photoabsorption at energies near resonances or the ionization threshold, the XFS is modified and is sensitive to the precise energy and bandwidth of the incident $x$-ray beam. In that case, the vacancy excitation and decay steps are coupled and described as a one-step, resonant inelastic scattering process. ${ }^{60}$ Numerous studies have been reported on this phenomenon, termed x-ray resonant Raman scattering (XRRS), and it continues to be an active field of research. ${ }^{37,38}$ Key early experiments include those of Sparks, ${ }^{61}$ Eisenberger et al., ${ }^{62}$ and Briand et al. ${ }^{63}$ The variations in x-ray spectral energies, intensities, and line shapes observed in those experiments are well explained by theoretical treatments $^{62,64,65}$ based on the resonant term of the Kramers-Heisenberg formulation of photon-scattering processes. ${ }^{3,45,60}$ There is much current interest in using XRRS to study electronic structure of solids ${ }^{66}$ and the dynamics of molecules excited at inner-shell 
resonances. ${ }^{67}$ Here we simply discuss XRRS from atoms, but the ideas are relevant to understanding experiments on complex targets.

Deslattes et al. ${ }^{39}$ and Cowan ${ }^{45}$ reported variations in Ar x-ray spectra excited below the K-ionization threshold, at the $1 \mathrm{~s} \square 4 \mathrm{p}$ resonance, and just above threshold which demonstrate XRRS features including linear dispersion, asymmetric line shapes, and line width narrowing. Those observations are consistent with the theoretical study of Tulkki ${ }^{68}$ showing the evolution of XRRS into $\mathrm{K} \square$ and $\mathrm{K} \square \mathrm{XFS}$ as the incident $\mathrm{x}$-ray energy is scanned through $\mathrm{Ar} \mathrm{K}$ edge resonances and threshold. To further characterize atomic XRRS, MacDonald et al. ${ }^{69}$ measured Xe $\mathrm{L}_{1,2}\left(\mathrm{~L}_{3} \mathrm{M}_{4,5}\right)$ and $\mathrm{L} \square_{2,15}\left(\mathrm{~L}_{3} \mathrm{~N}_{4,5}\right) \mathrm{x}$-ray spectra as the incident $x$-ray energy was scanned across the $L_{3}$ edge. Figure 16 shows a Xe $L \square_{1,2}$ spectrum, in which $3 d_{5 / 2}$ and $3 d_{3 / 2}$ electrons fill the $2 p_{3 / 2}$ vacancy. This spectrum was recorded $\square 27 \mathrm{eV}$ above the $L_{3}$ ionization energy $\mathrm{I}\left(2 \mathrm{p}_{3 / 2}\right)=4786.3 \pm 0.6 \mathrm{eV},{ }^{70}$ and the energies, intensities, and line shapes are representative of characteristic fluorescence. ${ }^{71}$ The excitation energy is sufficiently above threshold to use the two-step model. First, the photoelectron carries off the excess energy $\mathrm{h} \square-\mathrm{I}\left(2 \mathrm{p}_{3 / 2}\right)$, leaving a $2 \mathrm{p}_{3 / 2}$ hole state. Second, the radiative transition proceeds with $\mathrm{x}$-ray fluorescence lines appearing at

$$
I\left(2 p_{3 / 2}\right)-I\left(3 d_{5 / 2}\right)=4110.09 \pm 0.02 \mathrm{eV}
$$

and

$$
\mathrm{I}\left(2 \mathrm{p}_{3 / 2}\right)-\mathrm{I}\left(3 \mathrm{~d}_{3 / 2}\right)=4097.38 \pm 0.03 \mathrm{eV},
$$

where $\mathrm{I}\left(\mathrm{nl}_{\mathrm{j}}\right)$ is the subshell ionization energy. ${ }^{71}$ Symmetric Voigt line shapes are observed, with Lorentzian widths equal to the sum of the lifetime widths ${ }^{72}$ of a $2 p_{3 / 2}$ hole $(2.9 \mathrm{eV})$ and a $3 \mathrm{~d}$ hole $(0.5 \mathrm{eV})$ convoluted with a Gaussian instrumental width $(\square 1.6 \mathrm{eV})$. The relative intensities of the two lines are determined by the $\left[B d_{j}|r| 2 p_{3 / 2} \mid j=3 / 2,5 / 2\right.$ dipole matrix 
elements and multiplicity factors. The XFS in Fig. 16 does not depend on the precise energy or bandwidth of the incident $x$-rays, as long as the energy is far above threshold. ${ }^{73}$

Figure 17 shows the $\mathrm{Xe} \mathrm{L}_{3}$ absorption edge measured and fit by Breinig et al. ${ }^{70}$ to components consisting of $2 p \square n s, n=6,7,8, \ldots$ and $2 p \quad n d, n=5,6,7, \ldots$ Rydberg series leading to the $2 p \square[\beta, \square d$ continuum threshold. Spin-orbit quantum numbers are neglected here for simplicity. Also, as indicated in Fig. 17, the ns Rydberg series is relatively weak and will be neglected. The Rydberg and continuum components have been broadened to reflect the $2.9 \mathrm{eV}$ lifetime width of a $2 \mathrm{p}$ hole and strongly overlap. In XRRS theory, ${ }^{60}$ the Rydberg and continuum excitations indicated in Fig. 17 participate as intermediate states in an inelastic-scattering process:

$$
\mathrm{Xe}+\square(\text { in) } \square\{\mathrm{Xe}[2 \mathrm{p}] 5 \mathrm{~d}, 6 \mathrm{~d}, 7 \mathrm{~d}, \ldots, \square \mathrm{d}\} \square \mathrm{Xe}[3 \mathrm{~d}] 5 \mathrm{~d}, 6 \mathrm{~d}, 7 \mathrm{~d}, \ldots, \square \mathrm{d}+\square \text { (out) }
$$

where [nl]n'l' represents a state with a vacancy in the nl subshell and an electron in the n'l' subshell and \{\} indicates intermediate states. A Rydberg series of bound final states is produced: [3d]nd, $n=5,6,7, \ldots$, with a [3d] $[\mathrm{d}$ continuum state at the series limit, i.e., the same final state as in the $\mathrm{x}$-ray fluorescence process. For the bound final states, the scattered $\mathrm{x}$-ray spectrum displays peaks at fixed energy losses:

$$
E(\text { out })=E(\text { in) }-I([3 d] n d), n=5,6,7, \ldots .
$$

The bound-state peaks therefore display Raman-like linear dispersion as the incident $x$-ray energy is varied, and their lifetime widths are that of a $3 \mathrm{~d}$ hole $(0.5 \mathrm{eV})$ rather than a $2 p$ hole $(2.9 \mathrm{eV})$. This is the origin of the use of XRRS spectra to study inner-shell electronic structure at higher resolution than the inner-shell lifetime. ${ }^{66}$ Of course, the bandwidths of the incident x-ray beam and of the x-ray spectrometer must be narrow to take advantage of this effect. The distribution over intermediate states depends on the precise energy of the incident $x$-rays and will be modified by convolution over the incident bandwidth. ${ }^{68}$ Similarly, structure present in the scattered $\mathrm{x}$-ray spectrum will be broadened by the spectrometer bandwidth. 
The distinguishing feature in XRRS spectra associated with the [3d] $[\mathrm{d}$ continuum final state is that at incident $x$-ray energies in the range from below the $2 p$ ionization threshold to several lifetime widths above threshold, there is insufficient energy to fully develop the $x$ ray fluorescence peak. This is a simple consequence of energy conservation. ${ }^{68}$ How can the continuum final state be observed "below threshold"? Richtmyer et al. ${ }^{74}$ showed that the inner-shell lifetime width broadens the continuum edge jump into an arctangent shape extending below the nominal ionization energy, as shown in Fig. 17. LeBrun ${ }^{75}$ showed how energy-conservation arguments can explain the main features observed in XRRS spectra. In the inelastic-scattering picture, the threshold for producing a $3 d_{5 / 2}$ hole is the ionization energy, $676 \mathrm{eV}$, which is far below the $2 \mathrm{p}$ threshold. However, the cross sections for photon-scattering processes are relatively small in the absense of resonant intermediate states. The cross sections increase many orders-of-magnitude when the incident photon energy approaches the $2 p$ resonance and threshold region., ${ }^{3,60}$

The $\mathrm{Xe} L \square_{1,2} \mathrm{x}$-ray spectra recorded as the incident $\mathrm{x}$-ray energy was scanned across the $L_{3}$ resonances and threshold are shown in Fig. 18 in a perspective plot. The first eight spectra, recorded at incident energies from $12.8 \mathrm{eV}$ to $2.5 \mathrm{eV}$ below threshold, are plotted overlapping each other in Fig. 19 to show how the lineshape develops with increasing energy. As energy is increased above threshold, the peak positions remain fixed at the characteristic energies and the Lorentzian tails on the high-energy sides increasingly develop. The bandwidths of the incident x-ray beam $(\square 2 \mathrm{eV})$ and of the $x$-ray spectrometer $(\square 1.6 \mathrm{eV})$ blended the contributions of bound and continuum states and limited the observation of detailed structure which is expected to be present. ${ }^{68}$ However, the weak remnant of the bound states can be seen in Fig. 18 dispersing away from the fluorescence peak above threshold. The variations with incident $x$-ray energy of the $\mathrm{Xe}$ 
$\mathrm{L}_{1,2} \mathrm{x}$-ray spectra in Figs. 18 and 19 and similar variations for $\mathrm{Xe} L \square_{2,15}$ x-ray spectra ${ }^{69}$ are consistent within experimental resolution with XRRS features calculated by Tulkki. ${ }^{68}$

In XRRS theory, ${ }^{60}$ the cross sections for various final states are, in principle, sensitive to interference among the intermediate-state excitation amplitudes. In practice, atomic XRRS spectra have been modeled using the independent electron approximation in which interference effects are neglected. ${ }^{45,68,75}$ In this approximation, there is a one-to-one correspondence between the final-state cross sections and the lifetime-broadened resonant shapes of the intermediate states indicated in Fig. 17. The implication is that XRRS can be used essentially to measure a high-resolution absorption spectrum. ${ }^{66}$ However, in the context of solid samples, Carra et al. ${ }^{76}$ emphasized that XRRS spectra are sensitive to many-electron effects and must be interpreted with caution. In the case of radiationless decay in atoms, which involves two continuum electrons (photoelectron and Auger electron), post-collision interaction and relaxation effects are relatively strong. ${ }^{60}$ Armen et al. $^{77}$ recently reported an experimental and theoretical study of Auger resonant Raman scattering across the $\mathrm{Xe} \mathrm{L}_{3}$ edge, the radiationless analog to the XRRS study of MacDonald et al. ${ }^{69}$ Inclusion of relaxation and interference among intermediate-state amplitudes is required to explain the variations of Auger final-state intensities with excitation energy. ${ }^{77}$ Similar effects have been calculated and measured in Auger resonant Raman scattering at the Ar K-edge. ${ }^{78,79}$

Breinig et al. ${ }^{70}$ also measured the $\mathrm{Kr} \mathrm{K}$ absorption edge and fit it to the form of a $1 \mathrm{~s}$ $\mathrm{np}, \mathrm{n}=5,6,7, \ldots$ Rydberg series and ionization threshold. Schaphorst et al..$^{80}$ remeasured the $\mathrm{Kr} \mathrm{K}$ edge and found that calculated absorption cross sections for the Rydberg states and continuum edge underestimate the measured total cross section. To investigate XRRS at the $\mathrm{Kr} \mathrm{K}$ edge, we used the double-crystal $\mathrm{x}$-ray spectrometer shown in Fig. 15 to 
measure $\mathrm{K} \square_{1,2} \mathrm{X}$-ray emission spectra. A selection of the measured spectra is shown in Fig. 20. Asymmetric line shapes are observed at incident energies below threshold due to truncation of the fluorescent peaks, just as observed in Fig. 19 for $X e L_{1,2}$ XRRS spectra. Using $\mathrm{Si}(111)$ crystals, the bandwidths of the incident $\mathrm{x}$-ray beam ( $\square 4.7 \mathrm{eV})$ and of the spectrometer $(\square 2.4 \mathrm{eV})$ were too large to resolve bound final states in the $\mathrm{K} \square_{1,2} \mathrm{x}$-ray spectra in Fig. 20. However, the method of Hämäläinen et al. ${ }^{66}$ was used to search for resonance and threshold effects by comparing the high-resolution $\mathrm{x}$-ray fluorescence yield (HRXY) with the total absorption cross section.

The HRXY is defined here as the relative intensity of emitted $\mathrm{x}$-rays in the spectrometer bandwidth centered on the $\mathrm{K}_{1}$ characteristic peak energy $(\square 12649 \mathrm{eV})$. The structure observed in a HRXY spectrum ${ }^{66,69}$ can be understood heuristically by analogy to the Xe $\mathrm{L} \square_{1,2}$ spectra in Fig. 18. The integral over emission energy of the 2D spectrum in Fig. 18 would give a 1D spectrum analogous to the absorption spectrum in Fig. 17. Note that the large resonant peak in Fig. 18, which corresponds to excitation of the Rydberg series, includes a clearly visible tail, the central energy of which disperses one-to-one with incident $x$-ray energy. As a function of incident energy, the intensity integrated over emission energy represents a Lorentzian with a natural width corresponding to the core-hole lifetime. With better resolution, we expect the integrated peak intensity to mirror the partial contributions indicated by the fit in Fig. 17. This raises two important points. First, the 2D spectra provide an experimental approach to disentangling contributions from different final states in the absorption spectrum and to verifying the assumptions made in model fits to absorption spectra such as in Fig. 17. Second, confining the fluorescence excitation yield to the characteristic fluorescence energy means that we do not observe the tails which display the large Lorentzian lifetime broadening of the core hole. As a result, the contribution of the tails does not appear in the HRXY, and the resonance and threshold structure is manifested at an apparently higher resolution. This is in contrast with the total absorption spectrum, 
which sums over all final states. Indeed, as shown in Fig. 21, the HRXY has a sharper onset and intensity spike in the resonance and threshold region than does the absorption cross section. In addition, at $\square 30 \mathrm{eV}$ above threshold, the HRXY appears to be insensitive

to multielectron excitation features in the absorption spectrum. ${ }^{80}$ Multielectron excitations give rise to satellite peaks in $x$-ray emission, ${ }^{39}$ which presumably are excluded from the fluorescence yield measured here at the $\mathrm{K} \square_{1}$ peak energy. We conclude that by selecting a narrow band of final states, the HRXY gives information on resonance and threshold structure which complements total absorption cross section studies. More detailed information on atomic XRRS will result from future studies using higher resolution of both the incident and emitted $\mathrm{x}$-rays.

\section{X-ray polarization anisotropy of aligned molecules}

The atoms or molecules present in the target gas cell of the x-ray spectrometer in Fig. 14 are randomly oriented in space. However, x-ray absorption will generally produce an anisotropic state due to the transfer of angular momentum from the absorbed photon and due to its directionality and polarization. The anisotropy of the excited state will be reflected in the polarization and angular distribution of emitted x-rays. ${ }^{82,83}$ Gel'mukhanov and Mazalov ${ }^{84}$ predicted that excitation of molecules near threshold to states of specific symmetry will result in polarized $\mathrm{x}$-ray fluorescence. This effect was observed by Lindle et al. $^{48}$ in $\mathrm{Cl} \mathrm{K-V}$ resonant $\mathrm{x}$-ray fluorescence from $\mathrm{CH}_{3} \mathrm{Cl}$ and was followed by several experimental studies in which the x-ray spectrometer of Fig. 14 was rotated to measure either the polarization ${ }^{48-50,52-54}$ or angular distribution ${ }^{51,55,56}$ of emitted $x$-rays.

As discussed in the Section 6, the linear polarization of the incident $x$-ray beam and the polarization selectivity of the curved-crystal spectrometer in Fig. 14 both approach 100\% in many cases. Partial polarization of the incident beam and partial selectivity of the curved crystal can be treated, but for simplicity here we assume $100 \%$ for both. We also assume 
that $\mathrm{x}$-ray absorption and emission are well described as electric-dipole transitions, an approximation that was recently shown to break down in the case of resonant x-ray emission from $\mathrm{Cl}_{2}{ }^{52}{ }^{5} \mathrm{The}_{2}$ case will be discussed in the next section. Here we discuss experiments for which the electric-dipole approximation is expected to be valid.

X-ray absorption produces an anisotropic ensemble of core-excited atoms or molecules which has cylindrical symmetry around the polarization vector of the incident $x$-ray beam in Fig. 14. The photoexcited system is also symmetric with respect to reflection in the plane normal to the incident polarization vector. An excited system with these symmetry properties is said to be "aligned," and the symmetry properties are retained in the emitted x-ray patterns. $^{82,83}$ The angular distributions and polarizations of emitted x-rays can be characterized by measurements of $\mathrm{x}$-rays emitted in the plane normal to the incident $\mathrm{x}$-ray beam, with their polarization vectors oriented either parallel or perpendicular to that plane. Defining $\square$ to be the angle of $x$-ray emission with respect to the incident polarization vector, the angular distributions for parallel and perpendicular emission polarization are

$$
I_{I I}(\square)=I_{0}\left\{1+R\left[3 \sin ^{2} \square-1\right]\right\}
$$

and

$$
\mathrm{I}_{\square}(\square)=\mathrm{I}_{0}\{1-\mathrm{R}\}
$$

where $\mathrm{I}_{0}$ is proportional to the total intensity emitted in all directions and summed over polarizations, and $R$ is the polarization anisotropy. The parameter $R$ characterizes the radiation pattern and relates measurements to theory. ${ }^{55,85}$ The symmetry properties of the emitted $\mathrm{x}$-ray pattern are contained in the above expressions. For example, $\mathrm{I}_{\square}$ is isotropic and $I_{I I}\left(0^{\circ}\right)=I_{\square}$, so the emitted $x$-rays are unpolarized at $0^{\circ}$ (along the incident polarization vector). The measurement goal is to determine the parameter $R$ for a given $x$-ray absorption and emission process. $R$ can be determined by measuring the angular 
distribution of the parallel component, $I_{I I}(\square)$, or by measuring $I_{I I}$ and $I_{\square}$ at fixed $\square=90^{\circ}$ where

$$
R=2 P /(3-P)
$$

and

$$
P=\left(I_{1 \mid}-I_{\square}\right) /\left(I_{1 I}+I_{\square}\right)
$$

is the degree of linear polarization. The expression for $I_{I I}(\square)$ shows that an intensity proportional to the total intensity $I_{0}$ is measured by $I_{I I}$ at the "magic angle"

$$
\square_{m}=\sin ^{-1}\left(3^{-1 / 2}\right) \square 35.3^{\circ} .
$$

With the curved-crystal spectrometer positioned in parallel orientation at the magic angle, the relative intensities of the peaks in the XFS are proportional to their total emission cross sections. Measurements of $\mathrm{R}$ and $\mathrm{I}_{0}$ for $\mathrm{x}$-ray emission are analogous to measurements of photoelectron anisotropy parameters $\square$ and angle-integrated cross sections $\square$ for photoionization processes. $^{86}$

A rigorous theory of polarization anisotropies of photon emission from aligned atoms and molecules has been developed by Fano and Macek $^{82}$ and by Greene and Zare. ${ }^{83} \mathrm{~A}$ two-step model is used, but angular momentum quantum numbers are treated explicitly. The formalism is typically applied to cases involving small numbers of well-defined quantum states, i.e., atomic transitions and rotationally-resolved molecular transitions. This approach was used to understand the small polarization anisotropy $R=0.07 \pm 0.03$ that was measured for $\operatorname{Ar} \mathrm{K} \square$ x-rays following 1s $\square$ 4p resonant excitation. ${ }^{55,56}$ Large and distinctly different $R$ values are expected for different final-state multiplet components, but the mulitplet splittings are small and unresolved in the x-ray spectra, which washes out the anisotropy. This situation appears to generally apply to resonant $\mathrm{x}$-ray spectra of atoms, that is, large $R$ values are washed out by averaging over unresolved final states. 
Experimental studies of $\mathbf{x}$-ray polarization anisotropies from small molecules are more feasible than for atoms, because the molecular field splits the valence electronic states by several $\mathrm{eV}$, so they are often resolvable in XFS. Electronic states and transition moments must reflect the symmetry properties of molecular geometries, which provides the basis for producing aligned molecules by resonant $\mathrm{x}$-ray absorption and symmetry-dependent polarization anisotropies in $\mathrm{x}$-ray emission. A simple, classical two-step model ${ }^{87,88}$ has been used to qualitatively understand molecular $\mathrm{x}$-ray polarization anisotropies ${ }^{51}$ and will be discussed here. However, resonant x-ray emission should be described as a onestep, resonant inelastic scattering process, i.e., as XRRS but with uniquely molecular features. Recently, rigorous theoretical treatments have been developed for polarization anisotropies in molecular XRRS based on the Kramers-Heisenberg formula. ${ }^{89,90}$

In addition to Rydberg-like excitations leading to the ionization threshold as in rare gas atoms, ${ }^{70}$ molecular x-ray absorption spectra display strong subthreshold resonances due to excitation of valence-like states. Perera et al. ${ }^{91}$ have studied the $\mathrm{Cl} \mathrm{K}$ absorption spectrum and $\mathrm{Cl} \mathrm{K-V} \mathrm{XFS} \mathrm{of} \mathrm{CF}_{3} \mathrm{Cl}$. The electronic states are labeled by the $\mathrm{C}_{3 \mathrm{v}}$ molecular point group symmetries. For example, the $\mathrm{Cl}$ K-shell electrons are in the $1 \mathrm{a}_{1}$ molecular orbital (MO), and two of the valence MOs observed in the K-V XFS (see Fig. 22) are labeled $7 e$ and $10 a_{1}$. A strong, well-resolved subthreshold absorption resonance $^{91}$ is assigned as the $1 a_{1} \square 11 a_{1}$ transition, where the $11 a_{1} M O$ is the lowest unoccupied $\mathrm{MO}$ of the molecular ground state. Transition dipole moments between states of particular symmetries are directed along specific directions with respect to the molecular framework. The $1 a_{1} \square 11 a_{1}$ absorption dipole moment points parallel to the molecular figure axis (the $\mathrm{C}-\mathrm{Cl}$ bond axis). The direction of the emission dipole moment depends on the symmetry of the valence MO which drops into the $1 a_{1}$ vacancy. Valence MOs of $a_{1}$ 
symmetry have emission dipole moments directed parallel to the figure axis, while those for MOs of e symmetry are perpendicular to the figure axis.

Parallel-polarized XFS excited at the $11 \mathrm{a}_{1}$ resonance and recorded at $0^{\circ}$ and $90^{\circ}$ emission angles ${ }^{51}$ are plotted in Fig. 22. The relative intensities of the $7 e$ and $10 a_{1}$ peaks vary strongly between the two emission angles, so they have very different $R$ values. The variations can be understood using the classical two-step model in which the absorption and emission dipole moments are replaced by classical dipole oscillators treated as rigidly attached to the molecular framework. The expected $R$ values are determined by the relative directions of the absorption and emission oscillators averaged over molecular orientations. $^{88}$ Defining $\square$ to be the ensemble average of the angle between the absorption and emission oscillators, the polarization anisotropy is given by

$$
R=\left(3 \cos ^{2} \square-1\right) / 5
$$

The $1 a_{1} \square 11 a_{1}$ absorption oscillator is parallel to the molecular figure axis, so molecules in the target gas cell with their figure axes more closely aligned with the polarization vector of the incident $\mathrm{x}$-rays are preferentially excited. $A \cos ^{2} \square$ distribution of figure axes with respect to the incident polarization vector describes the alignment of the excited system. The $10 \mathrm{a}_{1}$ $1 a_{1}$ emission oscillator is also parallel to the figure axis, so $\square=0^{\circ}$ and $R=2 / 5$. The $7 \mathrm{e}$ $1 a_{1}$ emission oscillator is perpendicular to the figure axis, so $\square=90^{\circ}$ and $R=-1 / 5$. The $R$ values predict that the $10 \mathrm{a}_{1} \mathrm{MO}$ has its maximum intensity at $90^{\circ}$, while the $7 \mathrm{e} \mathrm{MO}$ has its maximum at $0^{\circ}$. The $10 \mathrm{a}_{1}: 7 \mathrm{e}$ intensity ratios measured at $\square=0^{\circ}, 30^{\circ}, 60^{\circ}$, and $90^{\circ}$ for both parallel- and perpendicular-polarization are plotted in Fig. 23 and compared with predictions of the classical two-step model. While the perpendicular-polarized intensity ratio is isotropic, the parallel-polarized ratio varies by a factor of 6 . The parallel- and perpendicular- 
polarized XFS recorded at $\square=90^{\circ}$ in a separate experiment ${ }^{50}$ are also consistent with the predicted polarizations

$$
P=3 R /(2+R)
$$

which gives $1 / 2$ and $-1 / 3$ for the $10 a_{1}$ and $7 e$ transitions, respectively.

Large polarization and anisotropy effects are observed in XFS for the large $\mathrm{R}$ values which result for resonant absorption to states of well-defined symmetry. The sensitivity of polarized XFS to molecular symmetry is its most useful application, as demonstrated by experimental $^{53}$ and theoretical ${ }^{89,92}$ studies of $\mathrm{H}_{2} \mathrm{~S}$. Above threshold, degenerate continuum channels of different symmetries usually contribute, so the alignment of the vacancy state and, consequently, the $R$ values and observed polarizations and anisotropies of the XFS, are relatively weak. Nevertheless, in the case of valence photoionization of molecules, the small polarizations produced in VUV fluorescence have been measured and used to study photoionization dynamics. ${ }^{85,93}$ Precise measurements of the polarizations and anisotropies of XFS excited above threshold could similarly provide information on photoionization dynamics of inner shells.

\section{Nondipole x-ray resonant Raman scattering from molecules}

The electric-dipole approximation to photon-electron interactions in atoms and molecules is usually expected to be valid when the photon wavelength is large compared with the radius of the electron's orbit. This criterion is met for the case considered in the previous section of $\mathrm{XRRS}$ near the $\mathrm{Cl} \mathrm{K}$-edge by $\mathrm{CF}_{3} \mathrm{Cl}$ which contains a single $\mathrm{Cl}$ atom. The $\mathrm{Cl} \mathrm{K}$ ionization threshold $\square 2833 \mathrm{eV}$ corresponds to an x-ray wavelength $\square 4.4 \AA$, which is large compared with the radius $\mathrm{r}_{1 \mathrm{~s}} \square 0.032 \AA$ of a $\mathrm{Cl} 1$ s atomic orbital. ${ }^{52}$ Molecular $\mathrm{Cl}_{2}$, however, contains two equivalent $\mathrm{Cl}$ atoms separated by an internuclear distance $\square 2.0 \AA$, suggesting that the XRRS process will be sensitive to variations of the phase of the incident and emitted $x$-rays over the interatomic distance. ${ }^{52,94}$ This idea was confirmed experimentally 
and explained theoretically in an XRRS study of $\mathrm{Cl}_{2}$ that was recently reported by Mills et al. ${ }^{52}$ It was necessary to treat the full exponential factor of the photon-electron interaction in the Kramers-Heisenberg formula, which resulted in showing how the XRRS spectrum is sensitive to diffraction-like structure factors. ${ }^{52}$ The discussion here will be limited to the experimental observation of nondipole final states in the polarized XRRS spectra of $\mathrm{Cl}_{2}$. We also discuss XRRS spectra of $\mathrm{Br}_{2}$, for which the $\mathrm{K}$ ionization energy $013474 \mathrm{eV}$ corresponds to an $\mathrm{x}$-ray wavelength $\square 0.92 \AA$, compared with the internuclear distance $\square 2.3$ Å. Predictions for XRRS spectra of $\mathrm{Br}_{2}$ were also calculated by Mills et al. ${ }^{52}$

The previous section discussed how the symmetries of molecular electronic states and transition dipole moments are related to polarization anisotropies of XFS. Among the symmetry properties which characterize states of homonuclear diatomic molecules is inversion through the center of symmetry. The states are either symmetric and labeled $\mathrm{g}$ (for gerade or even parity) or antisymmetric and labeled $u$ (for ungerade or odd parity). A selection rule for electric-dipole transitions is that the parities of atomic or molecular states must change when a photon is absorbed or emitted. For a two-photon process such as XRRS, the dipole selection rule requires that the final-state parity be the same as the initialstate parity. This parity selection rule has been confirmed ${ }^{67,95,96}$ in the cases of XRRS spectra of $\mathrm{N}_{2}$ and $\mathrm{O}_{2}$, for which the x-ray wavelengths are $\square 30 \AA$ and $\square 23 \AA$, respectively, i.e., large compared with internuclear distances. Skytt et al. ${ }^{67,97}$ and Glans et al. ${ }^{96}$ discuss the case of $\mathrm{CO}_{2}$, which also has inversion symmetry. However, the parity selection rule applies to the symmetry of the product wavefunction, electronic times vibrational, and $\mathrm{CO}_{2}$ has both even-parity (symmetric stretch) and odd-parity (antisymmetric stretch) vibrational modes. Still within the electric-dipole approximation, parity-forbidden electronic levels can be populated via the antisymmetric stretch and result in interesting vibronic coupling effects 
in XRRS. ${ }^{97,98}$ For homonuclear diatomic molecules, however, only even-parity symmetric stretch modes are involved, so vibronic coupling cannot account for observations of parityforbidden electronic states.

The $\mathrm{Cl} \mathrm{K}$ absorption spectrum and polarized K-V x-ray emission spectra were recorded using the instrumentation in Fig. 14. The absorption spectrum is plotted in Fig. 24 and is similar to the spectrum analyzed by Bodeur et al. ${ }^{99}$ The characteristic XFS measured above the ionization threshold is plotted in the bottom of Fig. 25 and shows partiallyresolved peaks due to the $2 \square_{g}, 2 \square_{u}$, and $5 \square_{g}$ valence MOs. The above-threshold XFS is not sensitive to the parity selection rule, because the unobserved photoelectron can have the parity required to give the many-electron state allowed total parity in each case. The three occupied valence MOs are built from linear combinations of $3 p$ atomic orbitals on the two $\mathrm{Cl}$ atoms, and the unfilled $3 p$ subshells also results in an unoccupied $5 \square_{\mathrm{u}} \mathrm{MO}$. A strong resonance occurs in the absorption spectrum (Fig. 24) due to $1 \square_{g} \square \quad 5 \square_{u}$ and $1 \square_{u} \quad 5 \square_{u}$ transitions, where $1 \square_{\mathrm{g}}$ and $1 \square_{\mathrm{u}}$ are the symmetric and antisymmetric linear combinations, respectively, of $1 \mathrm{~s}$ atomic orbitals on the two $\mathrm{Cl}$ atoms. The $1 \square_{\mathrm{u}} \mathrm{5} \square_{\mathrm{u}}$ transition is dipole forbidden, but becomes significant in the $\mathrm{Cl}_{2}$ case for which the $\mathrm{x}$-ray wavelength is comparable to internuclear distance. ${ }^{52}$ The $\mathrm{x}$-ray emission spectrum recorded on the $5 \square_{\mathrm{u}}$ resonance with parallel polarization is plotted in the top of Fig. 25. A resonant elastic peak appears in which the molecule has been left in the $X^{1} \square_{g}{ }^{+}$ground state. The remaining peaks constitute an XRRS spectrum with bound final states described by configurations with holes in the valence MOs and a $5 \square_{u}$ spectator electron: $\left[2 \square_{g}\right] 5 \square_{u} A^{1} \square_{u},\left[2 \square_{u}\right] 5 \square_{u}$ $\mathrm{B}^{1} \square_{g}$, and $\left[5 \square_{g}\right] 5 \square_{u} C^{1} \square_{u}{ }^{+}$. Comparison with the above-threshold XFS shows that the XRRS peaks are shifted to lower energies, and the $\left[5 \square_{g}\right] 5 \square_{u} C^{1} \square_{u}{ }^{+}$configuration splits into three final states. ${ }^{52}$ The gerade final states, $X^{1} \square_{g}{ }^{+}$and $B^{1} \square_{g}$, are dipole allowed, but the 
parity-forbidden states, $\mathrm{A}^{1} \square_{\mathrm{u}}$ and $\mathrm{C}^{1} \square_{\mathrm{u}}{ }^{+}$, are also strongly populated. The XRRS transitions are also polarized, as shown in Fig. 26. Mills et al. ${ }^{52}$ calculated a perpendicularpolarized XRRS spectrum for $\mathrm{Cl}_{2}$ which describes the measured spectrum well. The ideas discussed in the previous section to understand polarized x-ray emission in the context of the electric-dipole approximation are inadequate to describe polarized XRRS from $\mathrm{Cl}_{2}$. The full exponential factors in the photon-electron interactions in the Kramers-Heisenberg formula are required and causes the polarization and angular distribution of the XRRS spectrum to depend on diffraction-like structure factors. ${ }^{52,94}$

The halogen molecules $\mathrm{F}_{2}, \mathrm{Cl}_{2}$, and $\mathrm{Br}_{2}$ are valence-isoelectronic, i.e., the occupied and lowest-unoccupied MOs are built on $2 p, 3 p$, and $4 p$ atomic orbitals, respectively. Mills et al. ${ }^{52}$ used this correspondence to show how XRRS spectra for the three molecules vary between the electric-dipole regime $\left(F_{2}\right)$ to the hard $x$-ray regime $\left(\mathrm{Br}_{2}\right)$. Thus, the dipoleforbidden $1 \square_{u} \square \square_{u}$ resonant absorption is predicted to be negligible for $F_{2}$, significant for $\mathrm{Cl}_{2}$, and equal to the dipole-allowed $1 \square_{\mathrm{g}} \square \mathrm{Qu}_{\mathrm{u}}$ resonant absorption for $\mathrm{Br}_{2}$. However, an important point made by Mills et al. ${ }^{52}$ is that absorption spectra are not sensitive to nondipole and structure-factor effects while the XRRS spectra are. As a follow-up experiment to the $\mathrm{Cl}_{2}$ study, $\mathrm{K}-\mathrm{V}$ x-ray emission from $\mathrm{Br}_{2}$ was recorded using the doublecrystal spectrometer in Fig. 15. The x-ray spectra excited above the $\mathrm{K}$ ionization threshold and on the subthreshold resonance ${ }^{100}$ are plotted in Fig. 27. At the higher x-ray energies involved, $\mathrm{Si}(111)$ crystals were not adequate to resolve the valence final states for detailed analysis as in the $\mathrm{Cl}_{2}$ case. Two partially resolved peaks were recorded in each spectrum. The larger peaks apparently contain unresolved transitions from valence MOs while the smaller peaks contain inner-valence transitions. ${ }^{101}$ The peaks recorded on resonance are 
narrower and shifted to higher energy, indicating XRRS effects, but the resolution is

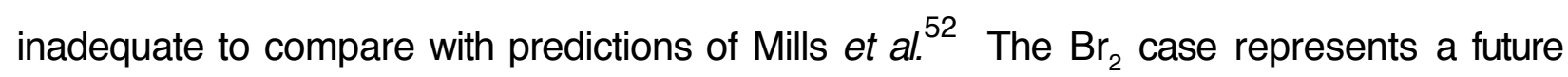
direction for development of both experiment and theory. Higher resolution and sensitivity are required to record XRRS spectra with well-resolved transitions from molecules containing higher- $Z$ atoms. Theoretical treatments will need to include spin-orbit interaction as well as nondipole and molecular geometry effects in resonant $x$-ray scattering.

\section{Acknowledgements}

We thank our Argonne coworkers R. W. Dunford, D. S. Gemmell, S. Hasegawa, M. Jung, and B. Krässig for their participation in recent experiments conducted at the Advanced Photon Source. We also thank B. Zabransky and the BESSRC staff (M. Beno, C. Kurtz, M. Engbretson, G. Jennings, G. Knapp, P. Montano, and C. Wiley) for support in conducting experiments at the APS. We thank P. M. Bergstrom, Jr., R. Bonham, J. P. J. Carney, M. Inokuti, L. LaJohn, and R. H. Pratt for guidance in understanding x-ray scattering from atoms, and we thank P. W. Langhoff, J. D. Mills, and J. A. Sheehy for explanations of resonant x-ray scattering from molecules. This work was supported by the U. S. Department of Energy, Office of Basic Energy Sciences under Contract No. W-31-109ENG-38. 


\section{References}

1. For a review, see for example B. Crasemann, in "Atomic Molecular and Optical Physics Handbook," edited by G. F. Drake (American Institute of Physics, Woodbury, NY, 1996), Chapter 60, p. 701.

2. P.M. Bergstrom, Jr. and R.H. Pratt, Rad. Phys. Chem. 50, 1 (1997).

3. J. J. Sakurai, "Advanced Quantum Mechanics" (Addison-Wesley, New York, 1967), Chapter 2.

4. J. H. Hubbell, W. Veigele, E. A. Briggs, R. T. Brown, D.T. Cromer, and R. J. Howerton, J. Phys. Chem. Ref. Data 4, 471 (1975); J. H. Hubbell, H. A. Gimm, and I. Overbo, J. Phys. Chem. Ref. Data 9, 1023 (1980).

5. C.T. Chantler, J. Phys. Chem. Ref. Data 24, 71 (1995); J. Wang, R.P. Sagar, H. Schmider and V.H. Smith, At. Data Nucl. Data Tables 53, 233 (1993); B.L. Henke, E.M. Gullikson and J.C. Davis, At. Data Nucl. Data Tables 54, 181 (1993).

6. J. Wang, R.O. Esquivel, V.H. Smith, Jr. and C.F. Bunge, Phys. Rev. A 51, 3812 (1995); H. Meyer, T. Müller, A. Schweig, Chem. Phys. 191, 213 (1995) and Acta Cryst. A 51, 171 (1995).

7. P. Eisenberger and P.M. Platzman, Phys. Rev. A 2, 415 (1970).

8. For a recent review, see M. J. Cooper, Radiat. Phys. Chem. 50, 63 (1997).

9. P. Coppens, "X-ray Charge Densities and Chemical Bonding" (International Union of Crystallography, Chester, England, 1997).

10. L. Kissel, R.H. Pratt, S.C. Roy, Phys. Rev. A 22, 1970 (1980).

11. L. Kissel, B. Zhou, S.C. Roy, S.K. Sen Gupta and R.H. Pratt, Acta Cryst. A 51, 271(1995).

12. E.O. Wollan, Phys. Rev. 37, 862 (1931).

13. D.R. Chipman and L.D. Jennings, Phys. Rev. 37, 862 (1963).

14. G.E. Ice, M.H. Chen and B. Crasemann, Phys. Rev. A 17, 650 (1978).

15. F. Smend and H. Czerwinski, Z. Phys. D 1, 139 (1986). 
16. M. Jung, R. W. Dunford, D. S. Gemmell, E. P. Kanter, B. Krässig, T. W. LeBrun, S. H. Southworth, L. Young, J. P. J. Carney, L. LaJohn, R. H. Pratt, and P. M. Bergstrom, Jr., Phys. Rev. Lett. 81, 1596 (1998).

17. R. Ali, I. Ahmad, R.W. Dunford, D.S. Gemmell, M. Jung, E. P. Kanter, P. H. Mokler, H. G. Berry, A. E. Livingston, S. Cheng, and L. J. Curtis, Phys. Rev. A 55, 994 (1997).

18. F. Biggs, L.B. Mendelsohn, J.B. Mann, At. Data. Nucl. Data. Tab. 16, 201 (1975).

19. P. M. Bergstrom, Jr, L. Kissel, R. H. Pratt, A. Costescu, Acta Cryst. A 53, 7 (1997).

20. A. N. Hopersky, V.A. Yavna and V. A. Popov, J. Phys. B 29, 461 (1996).

21. F. E. Low, Phys. Rev. 110, 974 (1958).

22. M. Gavrila, Phys. Rev. A 6, 1348 (1972); ibid 6, 1360 (1972).

23. P. M. Bergstrom, Jr., T. Suric, K. Pisk, and R.H. Pratt, Phys. Rev. A 48, 1134 (1993).

24. J. P. Briand, A. Simionovici, P. Chevalier, and P. Indelicato, Phys. Rev. Lett. 62, 2092 (1989).

25. V. Marchetti and C. Franck, Phys. Rev. A 39, 647 (1989); V. Marchetti and C. Franck, Phys. Rev. Lett. 65, 268 (1990).

26. S. Manninen, K. Hämäläinen and J. Graeffe, Phys. Rev. B 41, 1224 (1990).

27. V. Marchetti and C. Franck, Rev. Sci. Instrum. 59, 407 (1988).

28. J. Laukkanen, K. Hämäläinen, S. Manninen, V. Honkimäki, Nucl. Instrum. Methods A416, 475 (1998).

29. A comprehensive review is given in M. Inokuti, Rev. Mod. Phys. 43, 297 (1971); M. Inokuti, Y. Itikawa, J. E. Turner, Rev. Mod. Phys. 50, 23 (1978).

30. N. Watanabe, H. Hayashi, and Y. Udagawa, Bull. Chem. Soc. Jpn. 70, 719 (1997).

31. Some examples include: E.N. Lassettre and E.R. White, J. Chem. Phys. 60, 2460 (1974); A. Lahman-Bennani, A. Duguest, H.F. Wellenstein and M. Rouault, J. Chem. Phys. 72, 6398 (1980); R.S. Barbieri and R.A. Bonham, Phys Rev. A 44, 7361 (1991).

32. H. Hayashi, N. Watanabe, Y. Udgawa, and C.-C. Kao, J. Chem. Phys. 108, 823 (1998). 
33. K. Nishikawa and T. lijima, J. Chem. Phys. 87, 3752 (1987); T. Mitsuhashi and T. lijima, Chem. Phys. Lett. 109, 195 (1984).

34. R. A. Bonham, Phys. Rev. A 23, 2950 (1981).

35. N. Watanabe, H. Hayaski, Y. Udagawa, S. Ten-no, S. Iwata, J. Chem. Phys. 108, 4545 (1998).

36. "X-Ray Spectra and Chemical Binding", edited by A. Meisel, G. Leonhardt, and R. Szargan (Springer-Verlag, New York, 1989).

37. "Resonant Anomalous X-Ray Scattering: Theory and Applications", edited by G. Materlik, C. J. Sparks, and K. Fischer (North-Holland, Amsterdam, 1994).

38. "Raman Emission by X-Ray Scattering", edited by D. L. Ederer and J. H. McGuire (World Scientific, River Edge, NJ, 1996).

39. R. D. Deslattes, R. E. LaVilla, P. L. Cowan, and A. Henins, Phys. Rev. A 27, 923 (1983).

40. P. L. Cowan, S. Brennan, R. D. Deslattes, A. Henins, T. Jach, and E. G. Kessler, Nucl. Instrum. Methods A246, 154 (1986).

41. S. Brennan, P. L. Cowan, R. D. Deslattes, A. Henins, D. W. Lindle, and B. A. Karlin, Rev. Sci. Instrum. 60, 2243 (1989). Note that Fig. 3 of this reference is mislabeled and should read "ratio of $\square$ to $\square$ polarized light".

42. P. L. Cowan, J. B. Hastings, T. Jach, and J. P. Kirkland, Nucl. Instrum. Methods 208, 349 (1983).

43. A. Henins, Rev. Sci. Instrum. 58, 1173 (1987).

44. B. P. Duval, J. Barth, R. D. Deslattes, A. Henins, and G. G. Luther, Nucl. Instrum. Methods 222, 274 (1984).

45. P. L. Cowan, in "Resonant Anomalous X-Ray Scattering: Theory and Applications" (Ref. 37), p. 449.

46. E. W. McDaniel, "Atomic Collisions: Electron and Photon Projectiles" (Wiley, New York, 1989), p. 46. 
47. G. Margaritondo, "Introduction to Synchrotron Radiation" (Oxford University Press, New York, 1988), p. 38.

48. D. W. Lindle, P. L. Cowan, R. E. LaVilla, T. Jach, R. D. Deslattes, B. Karlin, J. A. Sheehy, T. J. Gil, and P. W. Langhoff, Phys. Rev. Lett. 60, 1010 (1988).

49. D. W. Lindle, P. L. Cowan, R. E. LaVilla, T. Jach, R. D. Deslattes, R. C. C. Perera, and B. Karlin, J. Phys. C 9, 761 (1987).

50. D. W. Lindle, P. L. Cowan, T. Jach, R. E. LaVilla, R. D. Deslattes, and R. C. C. Perera, Phys. Rev. A 43, 2353 (1991).

51. S. H. Southworth, D. W. Lindle, R. Mayer, and P. L. Cowan, Phys. Rev. Lett. 67, 1098 (1991).

52. J. D. Mills, J. A. Sheehy, T. A. Ferrett, S. H. Southworth, R. Mayer, D. W. Lindle, and P. W. Langhoff, Phys. Rev. Lett. 79, 383 (1997).

53. R. Mayer, D. W. Lindle, S. H. Southworth, and P. L. Cowan, Phys. Rev. A 43, 235 (1991).

54. K. E. Miyano, U. Arp, S. H. Southworth, T. E. Meehan, T. R. Walsh, and F. P. Larkins, Phys. Rev. A 57, 2430 (1998).

55. S. H. Southworth, D. W. Lindle, R. Mayer, and P. L. Cowan, Nucl. Instrum. Methods B 56/57, 304 (1991).

56. S. H. Southworth, Nucl. Instrum. Methods B 87, 247 (1994).

57. M. Ramanathan, M. A. Beno, G. S. Knapp, G. Jennings, P. L. Cowan, and P. A. Montano, Rev. Sci. Instrum. 66, 2191 (1995).

58. G. S. Knapp, M. A. Beno, C.S. Rogers, C. L. Wiley, and P. L. Cowan, Rev. Sci. Instrum. 65, 2792 (1994).

59. T. Matsushita and H. Hashizume, in "Handbook on Synchrotron Radiation", Vol. 1, edited by E.-E. Koch (North-Holland, New York, 1983), p. 261.

60. T. Åberg and B. Crasemann, in "Resonant Anomalous X-Ray Scattering: Theory and Applications" (Ref. 37), p. 431.

61. C. J. Sparks, Jr., Phys. Rev. Lett. 33, 262 (1974). 
62. P. Eisenberger, P. M. Platzman, and H. Winick, Phys. Rev. Lett. 36, 623 (1976).

63. J. P. Briand, D. Girard, V. O. Kostroun, P. Chevalier, K. Wohrer, and J. P. Mossé, Phys. Rev. Lett. 46, 1625 (1981).

64. Y. B. Bannett and I. Freund, Phys. Rev. Lett. 34, 372 (1975).

65. J. Tulkki and T. Åberg, J. Phys. B 15, L435 (1982).

66. K. Hämäläinen, D. P. Siddons, J. B. Hastings, and L. E. Berman, Phys. Rev. Lett. 67, 2850 (1991).

67. P. Skytt, P. Glans, J.-H.Guo, K. Gunnelin, N. Wassdahl, and J. Nordgren, in "Raman Emission by X-Ray Scattering" (Ref. 38), p. 193.

68. J. Tulkki, Phys. Rev. A 27, 3375 (1983).

69. M. A. MacDonald, S. H. Southworth, J. C. Levin, A. Henins, R. D. Deslattes, T. LeBrun, Y. Azuma, P. L. Cowan, and B. A. Karlin, Phys. Rev. A 51, 3598 (1995).

70. M. Breinig, M. H. Chen, G. E. Ice, F. Parente, B. Crasemann, and G. S. Brown, Phys. Rev. A 22, 520 (1980).

71. T. Mooney, E. Lindroth, P. Indelicato, E. G. Kessler, Jr., and R. D. Deslattes, Phys. Rev. A 45, 1531 (1992).

72. M. Ohno and R. E. LaVilla, Phys. Rev. A 45, 4713 (1992).

73. To simplify the description of characteristic XFS, we neglect satellite features due to multi-electron excitations which have energy onsets above the primary threshold (see Ref. 39).

74. F. K. Richtmyer, S. W. Barnes, and E. Ramberg, Phys. Rev. 46, 843 (1934).

75. T. LeBrun, in "Raman Emission by X-Ray Scattering" (Ref. 38), p. 142.

76. P. Carra, M. Fabrizio, and B. T. Thole, Phys. Rev. Lett. 74, 3700 (1995).

77. G. B. Armen, S. H. Southworth, J. C. Levin, U. Arp, T. LeBrun, and M. A. MacDonald, Phys. Rev. A 56, R1079 (1997).

78. G. B. Armen, J. C. Levin, and I. A. Sellin, Phys. Rev. A 53, 772 (1996). 
79. T. LeBrun, in "X-Ray and Inner-Shell Processes", edited by R. L. Johnson, H. SchmidtBöcking, and B. F. Sonntag (American Institute of Physics, Woodbury, New York, 1997), p. 647.

80. S. J. Schaphorst, A. F. Kodre, J. Ruscheinski, B. Crasemann, T. Åberg, J. Tulkki, M. H. Chen, Y. Azuma, and G. S. Brown, Phys. Rev. A 47, 1953 (1993).

81. W. Heitler, "The Quantum Theory of Radiation", 3rd edition (Dover, New York, 1984), p. 204.

82. U. Fano and J. H. Macek, Rev. Mod. Phys. 45, 553 (1973).

83. C. H. Greene and R. N. Zare, Ann. Rev. Phys. Chem. 33, 119 (1982).

84. F. Kh. Gel'mukhanov and L. N. Mazalov, Opt. Spectrosc. (USSR) 42, 371 (1977).

85. J. A. Guest, K. H. Jackson, and R. N. Zare, Phys. Rev. A 28, 2217 (1983).

86. S. T. Manson and D. Dill, in "Electron Spectroscopy: Theory, Techniques, and Applications", Vol. 2, edited by C. R. Brundle and A. D. Baker (Academic Press, New York, 1978), p. 157.

87. P. P. Feofilov, "The Physical Basis of Polarized Emission" (Consultants Bureau, New York, 1961).

88. R. N. Zare, "Angular Momentum" (Wiley, New York, 1988).

89. J. D. Mills and P. W. Langhoff, in "Raman Emission by X-Ray Scattering" (Ref. 38), p. 169.

90. Y. Luo, H. Ågren, and F. Gel'mukhanov, Phys. Rev. A 53, 1340 (1996).

91. R. C. C. Perera, P. L. Cowan, D. W. Lindle, R. E. LaVilla, T. Jach, and R. D. Deslattes, Phys. Rev. A 43, 3609 (1991).

92. F. Gel'mukhanov and H. Ågren, Phys. Lett. A 185, 407 (1994).

93. E. D. Poliakoff, in "Raman Emission by X-Ray Scattering" (Ref. 38), p. 220.

94. Y. Ma, Chem. Phys. Lett. 230, 451 (1994).

95. P. Glans, K. Gunnelin, P. Skytt, J.-H. Guo, N. Wassdahl, J. Nordgren, H. Ågren, F. Kh. Gel'mukhanov, T. Warwick, and E. Rotenberg, Phys. Rev. Lett. 76, 2448 (1996). 
96. P. Glans, P. Skytt, K. Gunnelin, J.-H. Guo, and J. Nordgren, in "X-Ray and Inner-Shell Processes", edited by R. L. Johnson, H. Schmidt-Böcking, and B. F. Sonntag (American Institute of Physics, Woodbury, New York, 1997), p. 723.

97. P. Skytt, P. Glans, J.-H. Guo, K. Gunnelin, C. Såthe, J. Nordgren, F. Kh. Gel'mukhanov, A. Cesar, and H. Ågren, Phys. Rev. Lett. 77, 5035 (1996).

98. L. S. Cederbaum, J. Chem. Phys. 103, 562 (1995).

99. S. Bodeur, J. L. Maréchal, C. Reynaud, D. Bazin, and I. Nenner, Z. Phys. D 17, 291 (1990).

100. B. M. Kincaid and P. Eisenberger, Phys. Rev. Lett. 34, 1361 (1975).

101. L. Frost, A. M. Grisogono, E. Weigold, C. E. Brion, A. O. Bawagan, P. Tomasello, and W. von Niessen, Chem. Phys. 119, 253 (1988). 


\section{Figure captions}

1. Cross sections for the primary photoprocesses: photoabsorption $\left(\square_{\mathrm{PE}}\right)$, elastic scattering $\left(\square_{E L}\right)$, inelastic scattering $\left(\square_{I N E L}\right)$, and pair production $\left(\square_{P P}\right)$ for carbon and lead. (Data from Ref. 4.)

2. Feynmann diagrams for scattering amplitudes in a non-relativistic framework. For elastic scattering, $\square_{1}=\square_{2}$. (a) "Seagull" diagram representing first-order $\mathbf{A}^{2}$ term; (b) and (c) represent second-order $\mathbf{p} \cdot \mathbf{A}$ terms.

3. Experimental setup for measuring polarization-independent scattering cross sections from gases. The two $\mathrm{Si}(\mathrm{Li})$ detectors are placed $90^{\circ}$ apart azimuthally around the incident photon beam direction. The scattering angle, $\square=90^{\circ} \pm 3^{\circ}$, as defined by two collimators. The incident photon beam is defined by slits and its intensity measured by ion chambers. The incident beam is polarized along the $\mathrm{x}$ direction.

4. Typical scattering spectra in the vertical $(y)$ detector. The empty cell spectrum is featureless, indicating no window scattering. The two small features at $\approx 25 \mathrm{keV}$ correspond to fluorescence from In and Sn impurities in the detectors. The light shaded areas of each spectrum indicate the Monte-Carlo simulation of the Rayleigh line, and the darker areas correspond to the simulated Compton peak.

5. Spectrum for Compton scattering of $18 \mathrm{keV}$ incident photons on a He target detected at $90^{\circ}$ scattering angle. The experimental points are shown as solid circles with error bars. The Monte-Carlo simulation, incorporating the detector response function and the impulse approximation to the Compton profile, and the fit residuals (multiplied by 100) are also shown.

6. Spectrum for Compton + Rayleigh scattering at $9 \mathrm{keV}$ on a $\mathrm{Ne}$ target (filled solid). When multiplied by 100 , the effect of the kinematic cutoff of the K-edge $(8.130 \mathrm{keV})$ on the 1s Compton profile is evident in the data. The Si escape peak of the detector is also seen at $7.3 \mathrm{keV}$. These features are all included in the simulations. 
7. Simulations of the experimental response to the subshell-specific Compton profiles within the impulse approximation for scattering from the $1 s, 2 s$, and $2 p$ subshells. The kinematic cutoffs produce asymmetric low-energy tails.

8. Spectrum for Compton + Rayleigh scattering at $22 \mathrm{keV}$ on a $\mathrm{Ne}$ target for $90^{\circ}$ scattering angle (filled solid). The darker filled region is the result of the Monte-Carlo simulation for Compton scattering while the lighter filled region is the same for Rayleigh scattering. The fit to the data (solid line) represents the sum of these simulations. Similar fits are also shown in Fig. 4 for the other energies.

9. Detector layout for subshell-specific Compton scattering experiments. The incident $x-$ ray beam points into the page with the electric vector parallel to the axis of $\mathrm{Si}(\mathrm{Li}) 3$. The beam bisects the region filled with $\operatorname{Ar}$ gas between $\mathrm{Si}(\mathrm{Li}) 1$ and $\mathrm{Si}(\mathrm{Li}) 2$.

10. Time delay between arrival of photons in Detector 2 relative to Detector 1 . The "prompt" spike at $\mathrm{t}=0$ includes the "true" coincidences. The circulation time for a given electron bunch at the Advanced Photon Source is $\approx 3.6 \square \mathrm{s}$, giving rise to the broad envelope structure. The fine structure arises from the $\square 100 \mathrm{~ns}$ between filled buckets in the ring.

11. (a) Energy in Detector 2 vs. energy in Detector 1 after the sorting for events contained in the $t=0$ peak shown in Fig. 10. (b) Energy in Detector 3 vs. energy in Detector 1 after the sorting for events contained in the $t=0$ peak shown in Fig. 10. Intensities are shown on log scales in both (a) and (b).

12. Coincidence spectrum (trues = prompts - randoms) isolating scattering from the K-shell in Ar. Coincidences in Detector 1 are obtained with a cut on the Kप, $\square$ fluorescence in the other detectors as well as the timing cuts. In the region of the Compton peak, the "singles" spectrum, shown as a solid line for comparison, is noticeably narrower than the coincidence spectrum since it is dominated by scattering from the outer shells of the atom.

13. The coincidence spectrum is compared to the impulse approximation Compton profile from Ref. 18. Note that the onset of scattering is displaced from the incident energy, 40 
$\mathrm{keV}$, by the K-shell binding energy $(3.2 \mathrm{keV})$. These data have not been corrected for the detection efficiency cutoff at low energies.

14. Diagram of the instrumentation used for the measurement of the angular distribution of polarized $\mathrm{x}$-ray emission from atoms and molecules following excitation by tunable synchrotron radiation.

15. Diagram of a double-flat-crystal $x$-ray spectrometer used for high-resolution $x$-ray spectroscopy of atoms and molecules.

16. $\mathrm{Xe} \mathrm{L} \square_{1,2} \mathrm{x}$-ray emission spectrum recorded $\square 27 \mathrm{eV}$ above the $\mathrm{L}_{3}$ ionization threshold to show the appearance of characteristic fluorescence.

17. Xe $\mathrm{L}_{3}$ absorption edge and fit to Rydberg and continuum components, from Breinig et al. $^{70}$

18. Variation of $X e L \square_{1,2} x$-ray emission spectrum across the $L_{3}$ edge showing resonant Raman scattering and the onset of characteristic fluorescence.

19. Xe $L \square_{1,2} \mathrm{X}$-ray emission spectra recorded at incident $\mathrm{x}$-ray energies between $12.8 \mathrm{eV}$ and $2.5 \mathrm{eV}$ below the $L_{3}$ ionization threshold and plotted overlapping each other to show the evolution of lineshape with increasing energy.

20. Variation of $\mathrm{Kr} \mathrm{K} \square_{1,2} \mathrm{x}$-ray emission spectrum at excitation energies across the $\mathrm{K}$ edge.

21. Comparison of $\mathrm{Kr} \mathrm{K}_{1}$ high-resolution $\mathrm{x}$-ray fluorescence yield (dotted line) with the total photoabsorption cross section (thick solid line). The thin solid line shows the expected $(\mathrm{h} \square)^{-7 / 2}$ asymptotic dependence on $\mathrm{x}$-ray energy of the one-electron photoionization cross section, ${ }^{81}$ normalized to the high-resolution fluorescence yield. Structures in the absorption cross section due to [1s4p] and [1s3d] multielectron excitations are indicated. ${ }^{80}$

22. Parallel-polarized $\mathrm{Cl} \mathrm{K-V}$ x-ray emission from $\mathrm{CF}_{3} \mathrm{Cl}$ excited on resonance at emission angles $\square=0^{\circ}$ (top) and $\square=90^{\circ}$ (bottom). 
23. Angular dependence of the $10 \mathrm{a}_{1}: 7 \mathrm{e}$ emission intensity ratio measured using parallel polarization (open circles) and perpendicular polarization (closed circles). The solid curve is a theoretical prediction for parallel polarization based on the classical two-step model and has been normalized to the measurement at $\square=90^{\circ}$. The dashed curve simply connects the perpendicular-polarized measurements, for which no variation with angle is expected.

24. Photoabsorption spectrum measured at the $\mathrm{Cl} \mathrm{K}$ edge of $\mathrm{Cl}_{2}$. The ionization threshold and $5 \square_{u}$ subthreshold resonance are indicated.

25. $\mathrm{Cl} \mathrm{K-V}$ x-ray emission spectra from $\mathrm{Cl}_{2}$ recorded with parallel polarization and excited on the $5 \square_{u}$ resonance (top) and above the ionization threshold (bottom).

26. $\mathrm{Cl} \mathrm{K-V} \mathrm{x-ray} \mathrm{emission} \mathrm{spectra} \mathrm{from} \mathrm{Cl}_{2}$ excited on the $5 \square_{\mathrm{u}}$ resonance and recorded with parallel polarization (top) and perpendicular polarization (bottom).

27. $\mathrm{Br} \mathrm{K}-\mathrm{V}$ x-ray emission spectra from $\mathrm{Br}_{2}$ excited on the subthreshold resonance (top) and above the ionization threshold (bottom). 